This is the author accepted version. Readers should cite the published version.

Knight, S., \& Thompson, K. (2020). Developing a Text-Integration Task for Investigating and Teaching Interdisciplinarity in Science Teams. Research in Science Education. https://doi.org/10.1007/s11165-020-09937-7

As part of the Springer Nature SharedIt initiative, readers should be able to access a full-text view-only version of the paper via https://rdcu.be/b4wQU. All readers of your article via the shared link will also be able to use Enhanced PDF features such as annotation tools, one-click supplements, citation file exports and article metrics. 


\title{
Developing a Text-Integration Task for Investigating and Teaching Interdisciplinarity in Science Teams
}

\author{
Simon Knight, University of Technology Sydney, simon.knight@ uts.edu.au * Corresponding author \\ Kate Thompson, Queensland University of Technology, kate.j.thompson@qut.edu.au
}

\begin{abstract}
Integrating information from across multiple sources is an important science literacy skill that involves: identifying intra and inter-textual ties; modeling relationships between sources and claims; and evaluation of the claims made. Tasks that involve reading, interpreting and synthesizing multiple sources have been well explored particularly in the epistemic cognition literature. Interdisciplinarity is a growing area of interest in science education, in terms of the ways we induct students into interdisciplinary ways of thinking and working, including the synthesis of knowledge from across scientific disciplines. While interdisciplinary contexts frequently involve connecting multiple sources from different disciplines, how students complete these text-integration tasks has not been well investigated. This paper develops a model of interdisciplinary text-integration for science literacy, drawing on dimensions of epistemic cognition. We exemplify the application of this approach in a specific case of environmental science graduate students, drawing on student syntheses to illustrate how our approach can be used to differentiate between students' written syntheses.
\end{abstract}




\title{
Developing a Text-Integration Task for Investigating and Teaching Interdisciplinarity in Science Teams
}

\begin{abstract}
Integrating information from across multiple sources is an important science literacy skill that involves: identifying intra and inter-textual ties; modeling relationships between sources and claims; and evaluation of the claims made. Tasks that involve reading, interpreting and synthesizing multiple sources have been well explored particularly in the epistemic cognition literature. Interdisciplinarity is a growing area of interest in science education, in terms of the ways we induct students into interdisciplinary ways of thinking and working, including the synthesis of knowledge from across scientific disciplines. While interdisciplinary contexts frequently involve connecting multiple sources from different disciplines, how students complete these text-integration tasks has not been well investigated. This paper develops a model of interdisciplinary text-integration for science literacy, drawing on dimensions of epistemic cognition. We exemplify the application of this approach in a specific case of environmental science graduate students, drawing on student syntheses to illustrate how our approach can be used to differentiate between students' written syntheses.
\end{abstract}

Keywords: interdisciplinarity; synthesis; writing; text-integration; science teams; epistemic cognition.

\section{Introduction}

Literacy, including the abilities to comprehend rich multimedia, and effectively communicate through written texts, is key to learning, and full participation in society across age ranges (OECD, 2013; OECD \& Statistics Canada, 2000). When learning science, it is common to expect students to engage with multiple texts in order to come to a conclusion about a topic. Britt and Rouet (2012) developed a model to describe how people comprehend multiple documents. They note that in fact when engaging with sources students may engage with documents in varied ways. Specifically, some students take the claims from each source and add that to their model of the topic (i.e., treating knowledge as accumulative); while other students create a richer model that includes the inter-relationships between the sources, and the document information (e.g. provenance features). Thus Rouet (2006), echoed by Bråten, et al., (2011) suggest some key skills that are important in the context of dealing with multiple sources (particularly in rich-multimedia environments): integration of prior knowledge and across documents (including competing claims); sourcing of features that identify the provenance, genre, etc. of the information; and corroboration to check information across multiple sources.

In interdisciplinary science contexts, text integration is particularly interesting, and under-researched. Much of the limited research on learning to do interdisciplinary research focuses on ways of collaborating, sharing and translating knowledge from different disciplines in teams (see, for example, Bammer, 2013; Pennington, 2016; Pennington et al., 2016). The alignment of values, theory, epistemologies and methods are identified challenges to the practice of interdisciplinarity (Lélé \& Norgaard, 2005). Given that a common output for research teams is in written form, whether an article, policy recommendation, or research proposal, text integration is key to the successful synthesis of disciplinary perspectives and appropriate communication to stakeholders. In this paper we describe a synthesis writing task that was designed for graduate environmental science students. We draw on work in epistemic cognition and text integration tasks in order to develop an approach to analysis of student texts and demonstrate the utility of the approach for differentiating between students' written syntheses in order to provide feedback. The work provides a novel account of text integration tasks as an epistemic probe on interdisciplinarity in science contexts.

\section{Background}

\section{Text Integration as a Lens on Learning}

A task that allows us to investigate these processes is text-integration or synthesis writing, in which students construct representations of how multiple sources fit together in relation to a particular task or issue (Goldman et al., 2013). Synthesis can occur in a number of different types of task, including: integrating differing genres of texts targeted at a shared theme; integrating texts that contain agreements and contradictions on a shared theme; or integrating texts 
that each contain pieces of information regarding a particular theme, each contributing a part of the picture (Goldman et al., 2013).

Text integration tasks can thus occur across a range of scientific contexts. Indeed, the need to integrate texts is commonplace. On a day-to-day level, people are frequently required to integrate information, for example regarding health behaviors such as dietary advice from multiple sources of varied qualities. Many of these contexts have societal implications, for example, how people engage in such integration tasks has implications for their participation in public debates around concerns such as climate change action (Bråten et al., 2011). For these reasons text integration is receiving increased attention in writing research, as a higher-order learning activity in its own right (P. D. Klein \& Boscolo, 2016), but not yet in interdisciplinary science research. Alongside this attention, there is increasing recognition of the disciplinary nature of writing (P. D. Klein \& Boscolo, 2016). Despite this dual concern, a feature of text integration tasks that has been little explored is the nature of disciplinary context in text integration tasks.

The skills involved in written synthesis are not specifically addressed in the literature on interdisciplinary learning in science, although communication and integration are both identified as key skills for interdisciplinary researchers (for example, Borrego \& Newswander, 2010; Vogler et al., 2018). Boix Mansilla (2006) found that researchers described a dynamic picture of knowledge validation in interdisciplinary projects, focusing on (1) how the work relates to what researchers find valid in the disciplines involved; (2) how the work stands together as a coherent whole; and (3) how the integration advances the goals identified by researchers. In Borrego \& Newswander's study (2010), they found that humanities literature emphasised (solitary) integration skills, whereas engineering and science proposals tended to emphasise interpersonal skills to demonstrate integration. Klein (2008) is one of the few researchers who have mentioned the ability to locate pertinent information, compare and contrast different approaches, and generate a synthesis as key to interdisciplinary research practice.

\section{Text Integration as a Probe on Epistemic Features of Interdisciplinarity}

A body of work in the learning sciences has explored these abilities to comprehend and integrate information from across sources, specifically viewing these behaviours through the lens of epistemic cognition (see, for examples, Barzilai \& Eshet-Alkalai, 2015; Bråten, 2008; Bråten et al., 2011; Ferguson, 2014). This construct, epistemic cognition, describes the ways in which people think about the nature of knowledge and knowing, typically described in terms of beliefs regarding the justification for claims, the source of knowledge, its complexity, and certainty (Hofer \& Pintrich, 2002). This work typically asks students to summarise, or develop arguments, based on internet search or sources that are provided (typically of varied provenance), requiring them to integrate and evaluate claims often on socio-scientific topics such as climate change (see particularly, Ferguson, 2014). In this work, students' cognition around the certainty, simplicity, source, and justification of knowledge is seen as a key mediator for how sources are treated and made use of, assessed through knowledge-tests or constructed responses. In text integration tasks, the ways in which sources are drawn on may give insight into learning. As Britt and Rouet note: "Quality learning involves not just acquiring facts about a topic or a situation, but also gaining an awareness of how these facts come to be established (i.e., what sources they come from) and the degree of certainty (or caution) with which they must be taken." (Britt \& Rouet, 2012, p. 279). Thus, in line with Bråten et al. (2011), successful integration must involve consideration of sources and relations among them, but readers may well not understand this consideration, may fail to recognize relationships and inconsistencies between sources, and if they do recognize inconsistencies, they may not attempt to reconcile them (Stadtler \& Bromme, 2014). Moreover, in order to effectively apply strategies to deal with inter-textual ties (making connections between texts), students must have meta-strategic knowledge (an approach to combining several strategies) that informs their understanding of when to draw on multiple sources (for example, where sources provide partial information, or disagree), and how to do this effectively (for example, through careful consideration of the claims, their basis, and their source, rather than merely listing the multiple claims) (Barzilai \& Ka'adan, 2017).

Analyses of student syntheses across disciplines indicate that there are a number of strategies that students use that do not support the range of different qualities we seek in a synthesis (less-adaptive strategies). These include: sourcing from a limited set of sources; sourcing key points from sources but without building connections between sources; and restatement of claims from sources but without integration (see, Goldman et al., 2012; Solé et al., 2013). In recent work on literacy and epistemic cognition (Anmarkrud et al., 2014; Bråten et al., 2014) students were asked to produce written outputs, which were then scored for:

1. Presence of explicit or implicit sourcing (i.e. explicit reference to the source, or indirect reference such as "one article spoke of [specific detail]" but without direct use of source information);

2. References to trustworthiness of the source or information from that source (coding separately for negative and positive evaluations);

3. Finally, whether connections were made between content-source trustworthiness (for example, whether content was trusted more because of the properties of the document from which it was sourced). 
That research found that, approximately half of sourcing references were explicit (with the other half implicit) and students did not make reference to the full list of sources (approximately three of six references). In Bråten et al., (2011) the authors describe some key relationships between a model of multiple document comprehension, and epistemic cognition. For example, we would expect those people who have less adaptive perspectives on the 'simplicity' of knowledge to engage with multiple documents in a way that emphasizes simple over complex sources, and accumulation of facts over integration.

Adapting this work to interdisciplinary contexts, we suggest that prior work on epistemic cognition and sourcing in text integration tasks can inform our understanding of interdisciplinary synthesis production in science contexts. For example, in drawing on that prior work we might characterize text syntheses that adopt single-discipline perspectives as expressing a less adaptive view of the 'simplicity' of knowledge. These relations between interdisciplinary text syntheses and epistemic cognition can be conceptualized as indicated in Table 1 . We suggest that developing tasks to understand key epistemic concerns in interdisciplinary learning contexts connected to writing could be useful.

$\underline{\text { Table } 1 \text { Hypothesized Relationships Between Epistemic Cognition and Interdisciplinary Text Integration }}$

\begin{tabular}{l|l|l}
\hline $\begin{array}{l}\text { Facet of } \\
\text { cognition }\end{array}$ & Less adaptive - characterized by & More adaptive - characterized by \\
\hline Simplicity & $\begin{array}{l}\text { The adoption of single-discipline } \\
\text { perspectives }\end{array}$ & $\begin{array}{l}\text { The synthesis of claims from multiple } \\
\text { disciplinary perspectives. } \\
\text { Effective intra-textual synthesis }\end{array}$ \\
\hline Certainty & $\begin{array}{l}\text { The adoption of fixed "solutions". } \\
\text { Focus on models that express certainty in } \\
\text { their application }\end{array}$ & $\begin{array}{l}\text { The use of multiple sources to support claims. } \\
\text { The tendency to draw on a diversity of } \\
\text { disciplinary perspectives }\end{array}$ \\
\hline Source & $\begin{array}{l}\text { An emphasis on sources that are from } \\
\text { familiar disciplines } \\
\text { The inclusion of disciplinary perspectives } \\
\text { that are not appropriate to the problem } \\
\text { space }\end{array}$ & $\begin{array}{l}\text { An emphasis on source characteristics } \\
\text { appropriate to ange of disciplines } \\
\text { Clear differentiation between source qualities } \\
\text { and the potential of disciplines to speak to } \\
\text { particular aspects of the problem space }\end{array}$ \\
\hline Justification & $\begin{array}{l}\text { An emphasis on methods or authorities } \\
\text { from a familiar discipline. } \\
\text { Little attempt at triangulation }\end{array}$ & $\begin{array}{l}\text { The use of triangulation to support claims. } \\
\text { An analysis of argument and expertise from } \\
\text { multiple disciplinary perspectives }\end{array}$ \\
\hline
\end{tabular}

Interdisciplinary writing tasks can be developed by providing learners with texts from multiple disciplines, within a particular context in which they must work towards identifying a specific problem, using a shared vocabulary, and set of resources to develop new understanding towards some ends, such as a policy recommendation or research proposal. In the rest of the paper, we describe a particular implementation of this interdisciplinary task design. We then draw on prior work in epistemic cognition and text integration tasks to develop a novel analytic approach for identifying relations between features in text-integration artefacts (i.e., written syntheses), and dimensions of epistemic cognition. And apply it to. Set of texts produced by graduate environmental science students participating in a text integration task. The work provides a novel account of text integration tasks as an epistemic probe on interdisciplinarity in science contexts.

\section{Methods}

\section{Participants and Ethics}

The Summer School was offered over two weeks for PhD students in July 2016, and 2017 at the University of Texas at El Paso (Thompson et al., 2017). Summer school participants were selected based on a variety of measures including their disciplinary background (including environmental science and engineering; archaeology; bioengineering; urban management; ecosystem science and sustainability; agriculture and biological engineering; agricultural economics; water science and management; water resources) and interest in interdisciplinary science. The research was determined to be exempt from full IRB Review. Participants were able to participate in the Summer School without being part of the research study, and were able to withdraw from the study (or from specific parts of the data collection) at any time. All participants consented to participation. In total thirteen graduate students (six males, seven females) participated 
in 2016, and thirteen in 2017 (five males and eight females). One student each year chose not to complete the writing task, and 24 texts were considered for this analysis.

\section{Materials and Procedure}

The Graduate Summer School (Thompson et al. 2017) was designed by a team of researchers from the environmental sciences and learning sciences and has been applied in multiple institutions (Thompson et al., 2016). The Summer School builds on a model for integrating knowledge across disciplines (Pennington, 2016) that combines theories from the learning, social, and organizational sciences regarding how visual externalization of mental models enables complex reasoning in groups (model-based reasoning). Over ten days, students engaged in a variety of tasks designed to focus on key elements of interdisciplinary socio-scientific research: collaborative processes and individuals' roles within a group; epistemic beliefs; production of visual representations; systems thinking; and leadership. Other skills were woven through the ten days, including reflective practice, identification of stakeholders, and written synthesis, the focus of this paper. Students were asked to write a synthesis of three articles, on a shared theme (the food-energywater nexus), but each from a different disciplinary context. The students were asked to draft syntheses through the workshop (on day 3 , with opportunities to redraft on days 5 and 8), with a final submission at the end of the event (the artefact reported in this paper).

\section{Analysis}

To analyze the synthesis texts, we identified features of the text that align with the high-level constructs indicated in Table 1. To do this, a rubric was developed to score the syntheses on five dimensions. In prior work by Goldman et al., (2013) essays were segmented into "idea units" that were mapped to their source texts and sentences, with an indication of its status as a summary. In a further formalized approach, Sorumen, Heinström, Romu and Turunen (2012) provide a detailed flowchart for the analysis of sourcing in source-based writing tasks. In that model, each sentence is assigned to a source document, with transformations categorized at both a paragraph and source level. However, these approaches are not readily applicable to an analysis in which it might be expected that students draw together information from multiple sources in a single sentence. They also do not provide further insight into the specific ways in which texts are woven together, nor the ways in which students do, or do not, evaluate the sources and their content. Although of course this information could be added as a categorization of text-transformation, and future work may adopt that approach, to provide a demonstration of the significance of synthesis to interdisciplinary learning, a simpler approach is adopted in this paper.

The rubric we adopt was based on our prior work investigating a text integration task (Knight \& Thompson, 2018) and is intended to be useable by an instructor or student without the need to undertake a time-consuming coding of individual sentences or idea units within a given text. The rubric builds on the literature cited above, drawing on the focus on: the specific content that students include in their texts; their use of explicit and implicit citation; the evaluation of the citations and the content drawn on; and the ways in which that information is synthesized (both summarizing individual sources and across multiple sources). The syntheses were analyzed with respect to their inclusion of topics or themes from the sources, intra- and inter-textual synthesis, evaluation, and sourcing (which articles were explicitly referred to). Each criterion of the rubric was scored on a 0-3 scale, with 0 indicating that the criterion was not addressed at all, and 3 that it was exemplary (for example, that topics from all three source documents were addressed).

1. Topic coverage: Ranging from the text giving superficial coverage of the topics $(0)$, through to clear coverage of topics from all three source texts (3).

2. Source inclusion: Ranging from text omitting reference to any source (0), through to reference to all three source texts (3).

3. Evaluation: Ranging from simple restatement of claims without consideration of source properties or the salience and plausibility of the claims made (0), through to a clear weighing up of the claims made between sources, analysis of disciplinary perspectives and differences, and drawing a conclusion (3).

4. Intra-textual synthesis: Ranging from simple restatement of claims from individual sources with no attempt at distilling key points or providing an overview of the text's claims (0), through to clear overview of each of the three texts with key claims present (3).

5. Inter-textual synthesis: Ranging from a total lack of integration between texts, for example, by discussing each text separately and in turn (0), through to an integration of the texts such that claims from each text can be identified inter-woven throughout the text (3). 
All 2016 texts were coded by both the first author and a research assistant, and agreement was calculated for each measure: topic (100\%), source, $(92 \%)$, evaluation (83\%), intra-textual (42\%), inter-textual (92\%). After discussion $100 \%$ agreement was achieved for each, and the research assistant coded all 2017 texts.

\section{Findings}

The table summarizes the text characteristics, by score, observed in this cohort group, showing in each row the characteristic split over the criteria (using a binary presence), a description and the number of student syntheses with the characteristics. This gives an indication of the range of responses, with each type described in further detail with reference to an example case from the group.

Table 2 - overview of synthesis response types

\begin{tabular}{rrrrrl}
\hline \hline Topic & Source & Evaluation & $\begin{array}{l}\text { Intra- } \\
\text { textual }\end{array}$ & $\begin{array}{l}\text { Inter- } \\
\text { textual }\end{array}$ & $\begin{array}{l}\text { Number of syntheses representing this type, brief description (and } \\
\text { student name used in the demonstration) }\end{array}$ \\
\hline 0 & 0 & 0 & 0 & 0 & 1: Summarises very generally; Samantha \\
1 & 0 & 0 & 1 & 1 & 5: Synthesises each topic without evaluation or sourcing; Anthea \\
1 & 1 & 0 & 1 & 0 & 4: Synthesises each paper separately without evaluation; Andrew \\
1 & 0 & 1 & 1 & 1 & 2: Synthesises and evaluates effectively without sourcing; Diane \\
1 & 1 & 1 & 1 & 0 & 2: Synthesises but without overall integration; Patrick \\
1 & 1 & 0 & 1 & 1 & 4: Synthesises overall without evaluation; Mark \\
1 & 1 & 1 & 1 & 1 & 6: Effective synthesis; Sandy \\
\hline
\end{tabular}

The results presented in Table 2 show that six of the 24 students included in the analysis produced an effective synthesis, meaning that they achieved a score of at least one in each category. An additional eight students achieved a score of at least one in all but one category (missing either the identification of the source (two students), an evaluation of the sources (four students) or the inter-textual description (two students)). Nine students were missing two of the key features of synthesis (in all cases an evaluation of the sources was missing, and in addition either an identification of the source or an inter-textual comparison). One student produced a general summary, only providing an inter-textual description. Most students identified the topic and produce an intra-textual description (23 students). The most common category that was missing was the evaluation of the sources (ten students included this).

In the following, we highlight seven students from the cohort who provide an example of the key features of each type of response, drawing on excerpts from their texts to demonstrate differences in how they completed the task, and how the features described in the analysis section help to differentiate between them. Each excerpt has been selected from the longer text produced to include one shared theme (precision agriculture). In presenting these excerpts, we also flag how the features described might be used to develop feedback to the students, and - where appropriate - which dimensions of epistemic cognition that feedback might particularly target.

Summary: Samantha was the only student to produce this less adaptive, high level coverage of all topics.

"As improvements are made in renewable energy, precision farming, and water management, sustainable practices are created and will inevitably impact the entire system. While the complexities of the individual systems continue to be investigated, dynamics within the systems and changes to the systems have significant impacts on the interconnection of the systems."

The excerpt illustrates that the synthesis provided considered high level commonalities, but without detail in topic coverage, evaluation, explicit or implicit use of sources. Because of the high level nature of the work, no identifiable inter/intra-textual ties could be readily identified. As can be seen in Table 2, feedback targeting the development of Samantha's synthesis would address all five features of the text-integration task.

Synthesis of each topic (no evaluation, no sourcing): Anthea submitted a text that synthesized the main ideas across texts and topics (referring to precision agriculture, energy, and water needs together), but without referring explicitly to the sources themselves, or evaluating the claims made.

"Energy-consuming machines have in many places replaced humans to plant, fertilize, harvest, and even water crops. Over time, as more machines have been used to produce and transport food, the amount of energy used from planting a seed to getting the produced food to someone's table has increased. However, the rise of precision agriculture can potentially help both increase the productivity of farms and decrease energy use. For instance, tractors using GPS guides can decrease the energy used by tractors by decreasing the number of overlapping passes that the tractor makes. This energy can come from many sources, some 
even involving the use of water sources, but energy production can also lead to pollution of water sources, thus contributing to water poverty. Additionally, large amounts of energy go towards cleaning groundwater that is contaminated with salt or water that has been polluted through various sources. Beyond these simpler connections, all three concepts are tied together by similar issues stemming from inherent ties to society, the economy, the environment, and technology."

As can be seen in Table 2, feedback targeting the development of this synthesis might focus on drawing explicitly on the sources, and critiquing the claims and their interrelations across the texts, for example, while the articles are drawn together they are not explicitly cited (e.g. the sentence "For instance, tractors using..." might cite the food article on precision agriculture). Moreover, evaluation would flow across the synthesis, for example, the food article presents a largely positive perspective on the potential of precision agriculture, but as Patrick's synthesis (below) notes "the implementation of this technology is not always an easy task as this is encompasses a social and economic aspect for the farmer to implement" (an evaluation not contained in the article itself), and this has implications for the wider potential of the technology in energy and water consumption, indeed related issues (of measurement in water policy) are noted elsewhere in the three papers, and could have been drawn on in developing critique.

Synthesis of each topic (no evaluation no inter-textual): In contrast, Andrew's text provides an example of a synthesis that was explicit in the identification of sources (by referring to the "first paper", etc.).

"First papers talks about precision agriculture how different technology is applied in agriculture to better use the resources and maximize agricultural production. This approach solves the unwanted consequences (erosion, soil and water quality deterioration) occurred while optimizing the crop yield. This paper somehow brings together nexus among the food, energy and water by talking about an approach that is designed to use less energy to maximize crop yield and trying to preserve the water quality."

In Andrew's writing, he summarized the claims from each paper independently in a list, drawing out intra but not inter-textual integration to cover all three topics, as the excerpt indicates. As can be seen in Table 2 , feedback targeting the development of Andrew's synthesis could address the use of evaluation and inter-textual ties in particular.

Synthesis (no sourcing): Dianne's work did not contain explicit reference to the sources from which the information was being drawn.

"In reading the presented literature on current issues in each discipline, three common issues emerged: 1) the affected parties 2) the role (and challenge) of big data, and 3) the role of (and need for) technological change in postponing catastrophe. [...]Almost all of the current problems addressed in the articles could have some solution if more data was available and researcher could therefore make more precise estimates, models, or scenarios. But at what cost? Big data can be intrusive. It can be expensive to collect. More importantly it must be modeled and used effectively to have any desirable outcome. Often FEW issues are limited by data and knowledge. Finding ways to access and use data accurately is a huge challenge for all three systems."

As the excerpt indicates, Dianne drew out key issues into a critical overview of the three themes, with particular attention to evaluation. As can be seen in Table 2, feedback to Diane could focus on sourcing, noting how the sources could be explicitly referred to in text, for example in the second paragraph, the first sentence ("Big data or more precise and frequent data..."), could in-line cite the food paper on precision agriculture.

Synthesis (no integration): Patrick's synthesis involved some critical discussion of the individual articles, but without synthesis of ideas across these articles.

"[3] As I review this paper once more, I found even though the technology is there to make more efficient crops and have higher yields, the implementation of this technology is not always an easy task as this is encompasses a social and economic aspect for the farmer to implement, in addition the technology can be a burden s the learning curve might be high for some farmers and in the end technology can be a burden if not used correctly. [...] Overall this paper can be linked to the previous two papers because one, the implementation of technology can make the farming process more efficient consuming less resources and increasing productivity which have led to increased expectations in the market to have a higher projection of yields once this technology is incorporated however the implementation of this technology might be cumbersome for the people working in the farms because they may view this technology as a replacement of field workers which have an impact on societal policies."

Patrick's text refers to the three sources separately (listed using [1-3]), using intra-textual synthesis to summarise the key points, but with very limited inter-textual synthesis (given in the final couple of sentences "Overall this paper can be linked..."). As the excerpt indicates, some evaluation is given, here discussing technology implementation (beyond discussion in the source piece). As can be seen in Table 2, feedback to Patrick could focus 
on drawing the three papers into a cohesive synthesized narrative (as in Diane's synthesis), rather than discussing the three papers in turn and tying them together only in that paper-by-paper discussion.

Synthesis (no evaluation): Mark's synthesis did not include explicit evaluation of the sources/content.

"The scale of the desired prediction places requirements on data types and amounts. For example, Lowenberg-DeBoer illustrates this with a discussion of variable rate fertilizer and the associated field data needed to make it actionable. Similarly, making monthly hydrological predictions requires (semi-)monthly data just as creating a spatial model at a specified scale requires a sample of observations within distinct spatial intervals of the scale for prediction. .... Building on this, Molle \& Mollinga point out that some institutions may even select arbitrary quantifications of human-environmental relations, again ignoring context, only to advance their own desired outcomes. Notably, quantifying resources like food, water, and energy for one place at one point in time can be difficult, even without making predictions, because these resources often exist in inter-connected environments. Energy, as Smil highlights, is incredibly difficult to predict because on one hand new resources (or technologies to extract them) emerge and on the other consumption patterns change both on the whole and regionally. Therefore, whatever predictions are made, they will be constrained by data availability"

As the excerpt indicates, connections between the texts and their key issues are drawn out into a critical overview of the three themes. As can be seen in Table 2, feedback to Mark could focus on evaluation of the claims made, for example while Mark's synthesis (including this excerpt) focuses on the issues of measurement flagged across the papers, the critique of this focus - in both the synthesis and the papers - is that it may obfuscate the potential for imperfect data to be used for positive outcomes across the nexus.

Effective synthesis: Finally, Sandy's text provides an example in which all three sources were drawn on and noted explicitly, with clear use of inter and intra-textual synthesis to effectively develop a concise, specific, and relevant set of claims on the issues presented.

"Problems in measurement often arise due to subjective or overly complex methods, or because measurement is difficult or unfeasible to execute. For example, Lowenberg-DeBoer (2015) describes "precision agriculture" as one technique used to understand food production in developing nations. Precision agriculture employs novel technologies used to analyze agricultural variables (e.g., soil quality, yield, land use and modification) at the micro-scale. The benefits of this detailed analyses can include increase in yields, as well as minimizing the negative environmental impacts of farming. However, economic constraints restrict the collection of large datasets and data collection techniques are often difficult for farmers to easily implement themselves. Molle and Molligna (2003: 541) suggest that indicators used to measure water scarcity in the development sector, likewise, are difficult to achieve. They suggest that measures of water scarcity, which often combine multiple variables to create a sliding scale, tend to obscure the intricacies of local resource issues."

This text uses the claims within each article to provide a critical analysis of the issues, using related claims across the articles to flag core issues by drawing out inter-textual relationships. Although no source based critique was offered (for example, of authorial credibility), the text does engage in some evaluation of the claims.

\section{Discussion and conclusions}

This paper has argued that text-integration tasks provide an important authentic interdisciplinary task, and a key lens into interdisciplinary thinking and epistemic cognition. We provide excerpts that exemplify the way in which these skills are demonstrated through text features in synthesis tasks. These excerpts suggest that the relatively more and less adaptive approaches to synthesizing information from interdisciplinary sources in Table 1 can be identified. Across these examples we see cases in which few sources are used (such as the summary, Synthesis and Evaluation without sourcing and synthesis without integration in Table 2), indeed in one case suggesting a 'certainty' (the summary Table 2) based on the use of a single source. Additionally we see differences in the use of sources and their source characteristics. At the most basic some responses did not include source information suggesting a relative downgrading of its importance in understanding the construction of knowledge (and syntheses) (the summary, synthesis without evaluation or sourcing, and synthesis and evaluates without sourcing in Table 2). Finally, we observed differences in the justification of information drawn on, with some syntheses engaging in little evaluation of the claims made (synthesis without evaluation or sourcing; synthesis without evaluation; effective synthesis in Table 2).

Despite the identification of written synthesis as an important skill for interdisciplinary scientific research, particularly for communication and integration (e.g. Klein, 2008). there are few studies that investigate this skill in the context of interdisciplinary science graduate student training. This study has provided a suggested task and a way of assessing whether a student has achieved this skill, based on what we know more broadly about epistemic cognition 
and text integration. The rubric designed for this study was able to be applied by a research assistant, and the suggested implications of the various combinations of scores are of use for science education practitioners. In addition, it has provided an interesting perspective on interdisciplinary science and the various components and skills required for interdisciplinary practitioners.

The particular case described in this paper is an example of an interdisciplinary task to probe epistemic features in syntheses. It could be expanded to include, for example, irrelevant material and sources of more varied quality, alongside the multiple-disciplinary perspectives presented here. Further analysis is required to draw out general approaches to connecting features of interdisciplinarity, text-integration, and epistemic dimensions. A step towards analyzing these relationships would be to include an existing measure of epistemic cognition in the measures provided to science students, to investigate the relationship between interdisciplinary synthesis and these established measures in epistemic cognition. In addition, further analysis should establish concurrent validity for the text quality, to investigate first how these texts are assessed by experts (e.g. environmental scientists), and second how these assessments relate to other observed interdisciplinary behaviors. The nature of the task will of course play an important role in the ways in which syntheses are produced as well as the relationships between text features and dimensions of epistemic cognition. For example, tasks that involve making policy recommendations are likely to produce rather different outputs to those that involve creating an overview synthesis of content for a proposal or paper, or a summary for stakeholders.

The findings of this paper show that text integration tasks hold potential for insight into interdisciplinary thinking and communication. The creation of artefacts has been recognized as an important component in the practice of interdisciplinarity, to facilitate the connection of ideas and knowledge within teams. However, these artefacts are also important in the communication of those ideas to others outside the team. The development of this task has shown that synthesis writing, and its links to features of epistemic cognition - simplicity, complexity, identifying sources, and justification - provide opportunities for investigating models of interdisciplinary learning and collaboration.

\section{Acknowledgments}

Our thanks to the EmBERS researchers and students, Penny Wheeler and David Williamson Shaffer for their helpful conversations on an earlier draft of this work, and Sue Chapman for her assistance in analysis.

\section{References}

Anmarkrud, Ø., Bråten, I., \& Strømsø, H. I. (2014). Multiple-documents literacy: Strategic processing, source awareness, and argumentation when reading multiple conflicting documents. Learning and Individual Differences, 30, 64-76. https://doi.org/10.1016/j.lindif.2013.01.007

Bammer, G. (2013). Disciplining Interdisciplinarity: Integration and Implementation Sciences for Researching Complex Real-World Problems. In Disciplining Interdisciplinarity. ANU E Press.

Barzilai, S., \& Eshet-Alkalai, Y. (2015). The role of epistemic perspectives in comprehension of multiple author viewpoints. Learning and Instruction, 36, 86-103. https://doi.org/10.1016/j.learninstruc.2014.12.003

Barzilai, S., \& Ka'adan, I. (2017). Learning to integrate divergent information sources: The interplay of epistemic cognition and epistemic metacognition. Metacognition and Learning, 12(2), $193-232$. https://doi.org/10.1007/s11409-016-9165-7

Boix Mansilla, V. (2006). Assessing expert interdisciplinary work at the frontier: An empirical exploration. Research Evaluation, 15(1), 17-29. https://doi.org/10.3152/147154406781776075 
Borrego, M., \& Newswander, L. K. (2010). Definitions of interdisciplinary research: Toward graduate-level interdisciplinary learning outcomes. The Review of Higher Education, 34(1), 61-84. https://doi.org/10.1353/rhe.2010.0006

Bråten, I. (2008). Personal Epistemology, Understanding of Multiple Texts, and Learning Within Internet Technologies. In M. S. Khine (Ed.), Knowing, Knowledge and Beliefs (pp. 351-376). Springer Netherlands. http://www.springerlink.com/content/j664674514614405/

Bråten, I., Braasch, J. L. G., Strømsø, H. I., \& Ferguson, L. E. (2014). Establishing Trustworthiness when Students Read Multiple Documents Containing Conflicting Scientific Evidence. Reading Psychology, O(0), 1-35. https://doi.org/10.1080/02702711.2013.864362

Bråten, I., Britt, M. A., Strøms $\varnothing$, H. I., \& Rouet, J.-F. (2011). The role of epistemic beliefs in the comprehension of multiple expository texts: Toward an integrated model. Educational Psychologist, 46(1), 48-70. https://doi.org/10.1080/00461520.2011.538647

Britt, M. A., \& Rouet, J.-F. (2012). Learning with multiple documents: Component skills and their acquisition. In J. Kiby R. ,. \& M. Lawson J. ,. (Eds.), Enhancing the Quality of Learning: Dispositions, Instruction, and Learning Processes. Cambridge University Press.

Ferguson, L. E. (2014). Epistemic Beliefs and Their Relation to Multiple-Text Comprehension: A Norwegian Program of Research. Scandinavian Journal of Educational Research, $0(0), \quad 1-22$. https://doi.org/10.1080/00313831.2014.971863

Goldman, S. R., Lawless, K., \& Manning, F. (2013). Research and development of multiple source comprehension assessment. In M. A. Britt, S. R. Goldman, \& J.-F. Rouet (Eds.), Reading: From words to multiple texts. New York: Routledge (pp. 180-199). Routledge.

Goldman, S. R., Lawless, K., Pellegrino, J. W., \& Gomez, K. (2012). A Technology for Assessing Multiple Source Comprehension: An Essential Skill of the 21st Century. In M. Mayrath (Ed.), Technology-based assessments for 21st century skills: Theoretical and practical implications from modern research. Information Age Publishing (IAP).

Hofer, B. K., \& Pintrich, P. R. (2002). Personal Epistemology: The Psychology of Beliefs About Knowledge and Knowing. Routledge. 
Klein, J. T. (2008). Education. In G. H. Hadorn, S. Biber-Klemm, W. Grossenbacher-Mansuy, H. Hoffmann-Riem, D. Joye, C. Pohl, U. Wiesmann, \& E. Zemp (Eds.), Handbook of transdisciplinary research (Vol. 10, pp. 399-410). Springer.

Klein, P. D., \& Boscolo, P. (2016). Trends in Research on Writing as a Learning Activity. Journal of Writing Research, 7(3 (February 2016)), 311-350. https://doi.org/10.17239/jowr-2016.07.03.01

Knight, S., \& Thompson, K. (2018). Developing a Text-Integration Task for Investigating and Teaching Interdisciplinarity in Science Teams. In J. Kay \& R. Luckin (Eds.), 13th International Conference of the Learning Sciences: Rethinking Learning in the Digital Age. Making the Learning Sciences Count (Vol. 3, pp. 1453-1455). International Society of the Learning Sciences. https://drive.google.com/drive/u/1/folders/1wTHiZuSn8Wn84Q1WpAL-ihQ6SvaDWP17

Lélé, S., \& Norgaard, R. B. (2005). Practicing interdisciplinarity. BioScience, 55(11), 967-975. https://doi.org/10.1641/0006-3568(2005)055[0967:PI]2.0.CO;2

OECD. (2013). PISA 2015: Draft reading literacy framework. OECD Publishing. http://www.oecd.org/pisa/pisaproducts/Draft\%20PISA\%202015\%20Reading\%20Framework\%20.pdf

OECD, \& Statistics Canada. (2000). Literacy in the Information Age-Final Report of the International Adult Literacy Survey. OECD. http://www.oecd.org/edu/skills-beyond-school/41529765.pdf

Pennington, D. (2016). A conceptual model for knowledge integration in interdisciplinary teams: Orchestrating individual learning and group processes. Journal of Environmental Studies and Sciences, 6(2), 300-312. https://doi.org/10.1007/s13412-015-0354-5

Pennington, D., Bammer, G., Danielson, A., Gosselin, D., Gouvea, J., Habron, G., Hawthorne, D., Parnell, R., Thompson, K., Vincent, S., \& Wei, C. (2016). The EMBeRS project: Employing model-based reasoning in socio-environmental synthesis. Journal of Environmental Studies and Sciences, 6(2), $278-286$. https://doi.org/10.1007/s13412-015-0335-8

Rouet, J.-F. (2006). The Skills of Document Use: From Text Comprehension to Web-Based Learning (1st ed.). Routledge.

Solé, I., Miras, M., Castells, N., Espino, S., \& Minguela, M. (2013). Integrating Information: An Analysis of the Processes Involved and the Products Generated in a Written Synthesis Task. Written Communication, 30(1), 63-90. https://doi.org/10.1177/0741088312466532 
Sormunen, E., Heinström, J., Romu, L., \& Turunen, R. (2012). A method for the analysis of information use in sourcebased writing. Information Research: An International Electronic Journal, $17(4)$, n4. http://eric.ed.gov/?id=EJ995300

Stadtler, M., \& Bromme, R. (2014). The content-source integration model: A taxonomic description of how readers comprehend conflicting scientific information. Processing Inaccurate Information: Theoretical and Applied Perspectives from Cognitive Science and the Educational Sciences, 379-402. https://books.google.co.uk/books?hl=en\&lr=\&id=TAFzBAAAQBAJ\&oi=fnd\&pg=PA379\&dq=he+content $\%$ E2\%80\%93source+integration+model:+A+taxonomic+description+of+how+readers+comprehend+confli cting+scientific+information.\&ots=vmHfzaLdR1\&sig=nPG3OYsx2d7oktEV_cB6QUDEIUE

Thompson, K., Danielson, A., Gosselin, D., Knight, S., Martinez-Maldonado, R., Parnell, R., Pennington, D. D., Svoboda-Gouvea, J., Vincent, S., \& Wheeler, P. (2017). Designing the EMBeRS Summer School: Connecting Stakeholders in Learning, Teaching and Research. Proceedings of the 25th International Conference on Computers in Education.

Thompson, K., Gouvea, J. S., \& Habron, G. (2016). A Design Approach to Understanding the Activity of Learners in Interdisciplinary Settings: Environment and Diversity. Transforming Learning, Empowering Learners.

Vogler, J. S., Thompson, P., Davis, D. W., Mayfield, B. E., Finley, P. M., \& Yasseri, D. (2018). The hard work of soft skills: Augmenting the project-based learning experience with interdisciplinary teamwork. Instructional Science, 46(3), 457-488. https://doi.org/10.1007/s11251-017-9438-9 